\title{
Late onset myasthenia gravis: A review and report of 24
}

\section{cases}

\author{
Andrea L Porras-Yaurivilca ${ }^{1}$, Tulio E Bertorini' ${ }^{2 *}$, Victor J Duenas-Vicuna ${ }^{3}$ and William H Mays ${ }^{2}$ \\ ${ }^{1}$ Hospital Nacional Hipolito Unanue. Lima, Peru \\ ${ }^{2}$ Wesley Neurology, Memphis, TN, USA \\ ${ }^{3}$ Instituto Nacional de Ciencias Neurologicas, Lima, Peru
}

\begin{abstract}
Introduction: The objective of this study was to review the literature and analyze the clinical presentation and response to therapy of late onset myasthenia gravis (LOMG) in our center.

Methods: Previous reports of LOMG and the records of 24 cases of LOMG seen in our neuromuscular clinic were reviewed, and the demographic data, clinical presentations, and responses to therapy were studied. The definition of LOMG's age of onset varies, but we have defined the cases in our center as LOMG when symptoms first appear at 65 years of age or older.

Results: In our center, the age range was $65-82$ years--the male sex predominated in 16 patients $(67 \%)$ while 8 patients (33\%) were women; the presenting symptom was ocular in 12 patients (50\%), generalized (not confined to a specific muscle group) in 7 (29\%), and bulbar in 5 (21\%); this is similar to statistics reported previously. The most common comorbidities were diabetes, essential hypertension, and thyroid disease. The treatment consisted of acetylcholinesterase inhibitors (monotherapy) in two patients; prednisone plus acetylcholinesterase inhibitors were administered in 20 patients (83\%); 17 subjects (71\%) needed immunosuppression with azathioprine / mycophenolate mofetil (due to lack of response or for steroid sparing). Ten patients (42\%) in our center required plasma exchange or intravenous immunoglobulin infusions; thymectomy was performed in three cases, and one of these had a thymoma; otherwise, no patients experienced thymic hyperplasia.
\end{abstract}

Conclusions: As in the literature, we found LOMG to have a clear male predominance; ocular presentation was the most common manifestation. Clinically, antibody positivity and response to therapy in LOMG were similar to younger myasthenia patients (non-LOMG patients) as evidenced by previous studies. Thymoma was rare.

\section{Introduction}

Myasthenia gravis is an autoimmune disease of the neuromuscular junction caused by antibodies and autoreactive $T$ cells that attack the acetylcholine receptors (AChR) thus blocking and degrading these receptors with the formation of complement attack complexes [13]. Other pathogenic mechanisms include antibodies against postsynaptic proteins such as muscle-specific kinase (MuSK) [4], but in about $10 \%$ to $15 \%$ of cases, no antibodies have been demonstrated [5]; those cases are labeled sero-negative. Recently, antibodies against other neuromuscular junction proteins such as agrin and lipoprotein receptor-related protein 4 (LRP4) have been reported in double seronegative cases [6]. There are no reports of the incidence of these antibodies in LOMG.

Neuromuscular junction disorders affect approximately 100,000 people each year, and myasthenia gravis is the most common disease of the neuromuscular junction. Myasthenia is responsible for approximately $75 \%$ of these 100,000 patients, and it primarily affects young women $[7,8]$. Frequently these patients, particularly the younger ones, have thymic hyperplasia [7,9] and the HLAB8D3 antigen [9]. The disease can also occur in older persons with less sex predominance and a weaker association with HLAB8D3 [10].

An increasing incidence of myasthenia in older patients (LOMG) has been reported [10-14], but not all studies concurred with this finding [15], and the cutoff age for classification of LOMG varies from study to study making the assessment of incidence difficult $[10,12$ -
14,16-27]. Recognition of LOMG, however, is important; there are significant therapeutic implications regarding misdiagnoses [16-18]. There may be a decreased incidence of thymic enlargement in LOMG, and patients may not benefit from thymectomy $[19,20]$. Management of LOMG is further complicated by the advanced age of the population; these patients also tend to have more comorbidities and are on more maintenance medicines with complications to therapy; furthermore, they are usually worse surgical candidates [21]. Older patients have been reported to require less aggressive immunotherapy [22].

We reviewed the literature and performed a retrospective study of LOMG in our medical center using the cutoff age of onset at 65 to determine the clinical characteristics, sex predominance, presence of thymic hyperplasia, and response to and complications of therapy.

\section{Materials and methods}

The records of patients diagnosed with myasthenia gravis seen in our clinics from January, 2010 to March, 2015 were reviewed. A total of 98 cases were identified, and of these, 24 were 65 years old or greater

Correspondence to: Tulio E. Bertorini, MD, Wesley Neurology, 1211 Union Avenue, Suite 400, Memphis, TN 38104, USA, Tel: 901-725-8920; E-mail: tbertorini@aol.com

Key words: late onset myasthenia gravis, LOMG, myasthenia gravis, MG, thymic hyperplasia, thymoma

Received: March 31, 2018; Accepted: April 10, 2018; Published: April 13, 2018 
at the time of clinical presentation. The diagnosis of myasthenia gravis in all of these patients was made by clinical features, antibody titers, electrophysiology and edrophonium testing. We studied the incidences of race, sex, ages at onset, antibody presentations, and the presence of thymic pathologies. We also studied the responses to treatment, complications, and prognoses, and we compared the findings with those reported in the reviewed literature.

\section{Results}

We identified a total of $24 \mathrm{LOMG}$ patients out of 98 (24.4\%), and there was a male predominance of 16 males $(67 \%)$ and 8 females (33\%). A higher female incidence has been reported by others [23], but most of the literature reported male predominance in LOMG. The age range was 65 to 82 with mean age of onset at 68 . One patient was over 80 years of age. The initial presentation was ocular in 12 patients (15\%), generalized in $7(29 \%)$, and one patient presented with bulbar symptoms at age 82 (Figure 1). Eighteen (75\%) patients were AChR antibody positive, $3(12.5 \%)$ were MuSK antibody positive, and 3 (12.5\%) were double negative (Figure 2). All had generalized myasthenia during the evaluation. The most common comorbidities identified in our study were diabetes, essential hypertension, and thyroid disease. Thymoma was identified in one patient (4.2\%) which is lower than reported by others in LOMG [18]. No thymic enlargement was seen on computed tomography (CT) scans, and no thymic hyperplasia was seen on pathology reports after surgery in 2 patients $(8.3 \%)$ who underwent thymectomy selectively. Of the 24 patients, 3 died (mortality $12.5 \%$, higher than the reported overall mortality): 2 of sepsis and 1 of disseminated thymoma.

Treatment consisted of only acetylcholinesterase inhibitors in two patients. Prednisone was added in 20 (83\%); 18 (75\%) needed immunosuppression with azathioprine and mycophenolate mofetil due to lack of response or for steroid sparing; 10 (42\%) required plasma exchange or intravenous inmumoglobulins infusions (Figure 3) while remaining on steroids.

\section{Discussion}

We found an incidence of $24.3 \%$ of LOMG of the total number of myasthenia gravis patients seen in our center. This is similar to that reported in the literature [10-14]. There was clear male predominance (67\%); no sex predominance has been reported by others, but this is likely related to the cutoff age of 35 years old used to define LOMG [5,24]. The incidence of MuSK antibody positivity was $14.5 \%$ while others have reported an incidence to $23 \%$ to $38 \%[4,25]$. We found an incidence of double sero-negativity to be $12.5 \%$--this has been reported to be $10-15 \%$ in previous reports [5]. Others have reported an increased

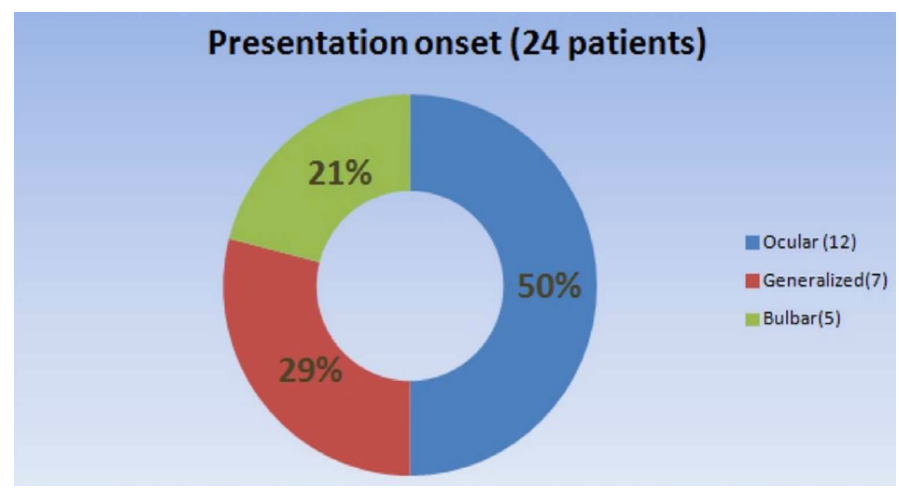

Figure 1. Presenting symptoms.

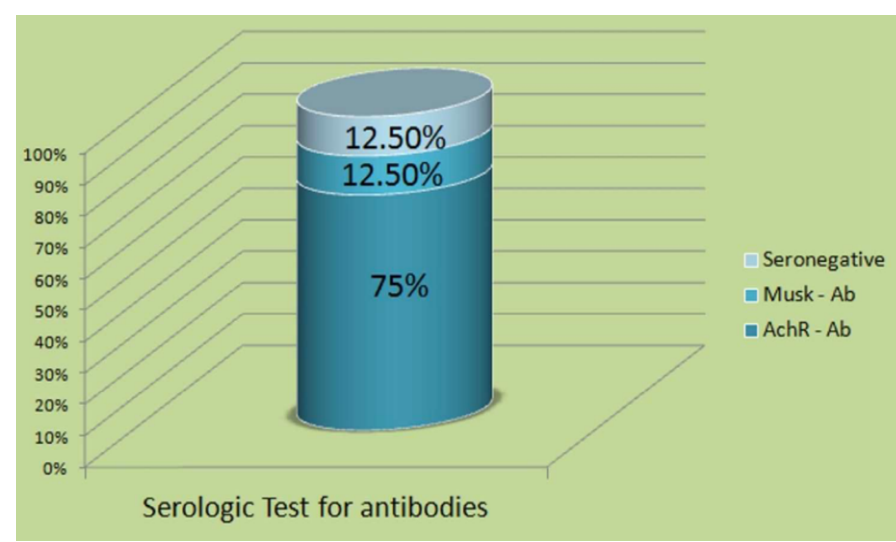

Figure 2. Antibody testing.

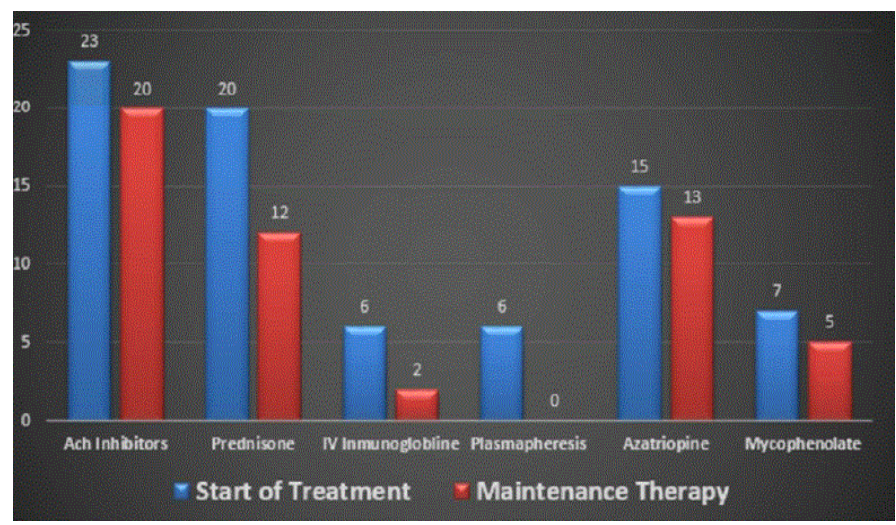

Figure 3. Treatment modalities.

incidence of anti-titin and anti-ryanodine receptors in LOMG $[28,29]$. we did not analyze this nor test for agrin or LRP4 antibodies.

The thymus gland has been implicated in the pathogenesis of MG, but we did not find thymic enlargement radiologically or hyperplasia pathologically in two patients who underwent thymectomy, and we found only one patient with thymoma.

It appears that LOMG is somewhat different from early onset MG because of male predominance, less incidence of thymic hyperplasia, and the lack of association with the HLAB8D3. There seem to be immunological differences between early and LOMG, and, in the latter, there is an association with the HLADRB $1^{\star} 15: 01$ antigen as cited in previous reports; association studies of the major histocompatibility complex (MHC) region detected association with the HLAA, MHC class 2, HLA8, and MHC class III [22]. This was not studied in our population.

The response to medical treatment in our cases was excellent and similarly has been reported $[13,26,30]$. Thymectomy has been shown to be safe and effective in the elderly while others have reported thymic hyperplasia in older patients $[19,20]$. A recent multi-center study clearly demonstrated the benefit of thymectomy in myasthenia gravis; in this study, however, there was no clear benefit when the procedure was performed in patients older than 50 years old [23,31,32].

In conclusion, LOMG has a clinical presentation similar to early onset myasthenia, but some differences exist including a lower incidence of thymic hyperplasia and a better response to therapy in LOMG patients. Confounders in this older population are complications with treatment due to age. We conclude that thymectomy could 
be considered in LOMG only if there is radiological evidence of an enlarged thymus on CT or MRI.

\section{Disclosure}

The authors of this paper have no financial interests to disclose.

\section{Acknowledgements}

Authors AP and VD completed much of the data gathering and composition of the text of the paper. TB contributed to the data gathering and intellectual supervision. WM performed much of the editing and clarification of the paper's primary conclusions.

\section{References}

1. Drachman DB (1994) Myasthenia gravis. NEJM 330: 1797-1810.

2. Huang GZ, Lo YC (2013) Correlation between acetylcholine receptor antibody levels and thymic pathology in myasthenia gravis; A review. Journal of Clinical Neuromuscular Disease 14: 2009-2117. [Crossref]

3. Engel AG, Arahata K (1987) The membrane attack complex of complement a the endplate in myasthenia gravis. ANNMY Acad Scil 505: 326-332. [Crossref]

4. Hoch W, McConville J, Helms S, Newsom-Davis J, Melms A, et al. (2001) Auto antibodies to the receptor tyrosine kinase MuSK in patients with myasthenia gravis without acetylcholine receptor antibodies. Nat Med 7: 365-368. [Crossref]

5. Vincent A, McConville J, Farrugia ME, Newsom-Davis J (2004) Seronegative myasthenia gravis. Semin Neurol 24: 125-133. [Crossref]

6. Richman OP (2015) The future of research in myasthenia. JAMA Neurology 27: 812814. [Crossref]

7. Vincent A1, Palace J, Hilton-Jones D (2001) Myasthenia gravis. Lancet 357: 21222128. [Crossref]

8. Oosterhius HJGH (1997) Clinical aspects and epidermiology in: Myasthenia gravis. Gromingen Neurological Pres 17-48.

9. Compton DAS, Vincent A, Newson-Davis J, Batchelor JR (1980) Clinical pathological HLA antigen and immunological evidence for disease heterogeneity in myasthenia gravis. Brain 103: 579-581. [Crossref]

10. Aarli JA (1999) Late-onset myasthenia gravis: a changing scene. Arch Neurol 56: 2527. [Crossref]

11. Lavrnic D, Basta I, Rakocevic-Stojanovic V, Stevic Z, Peric S, et al. (2013) Epidemiological study of a adult-onset myasthenia gravis in the area of Belgrade (Serbia) in the period of 1979-2008. Neuroepidermiology 40: 190-194. [Crossref]

12. Aarli JA (2008) Myasthenia gravis in the elderly: Is it different? Ann N Y Acad Sci 1132: 238-243. [Crossref]

13. Alkhawajah NM1, Oger J (2013) Late-onset myasthenia gravis: a review when incidence in older adults keeps increasing. Muscle Nerve 48: 705-710. [Crossref]

14. Aragones JM, Bolibar I, Bonfill X, Bufill E, Mummany A, et al. (2003) Myasthenia gravis. A higher than expected incidence in the elderly. Neurology 60: 1024-1026. [Crossref]
15. Pedersen EG, Hallar J, Hanseuk, Jensen PE, Gaist D (2013) Late onset myasthenia not on the increase: A nationwide register in Denmark. Eur J Neurol 20: 209-314. [Crossref]

16. Vincent A, Clover L, Buckley C (2003) Evidence of under diagnosis of myasthenia gravis in older people. Journal Neurol Neurosurg Psychiatry 74: 1105-1108.

17. Levintoglu A, Kuruoglu HR (2009) Late onset myasthenia gravis masquerading as a stroke. Jour Neurol Sci 242-247.

18. Aarli JA (2008) Myasthenia gravis in the elderly: Is it different? Ann N Y Acad Sci 1132 238-243. [Crossref]

19. Usawa A, Kaswaguichi N, Kanai T (2015) Two-year outcome of thymectomy in nonthymatous late onset myasthenia gravis. J Neurol 262: 1019-1023.

20. Tsuchida M, Yamato Y (1999) Efficacy and safety of extended thymectomy for elderly patients with myasthenia gravis. Ann Thorac Surg 67: 1563-1567. [Crossref]

21. Punga AR, Sawada M, Stalberg EV (2008) Electrophysiological signs and the prevalence of adverse effects of acetylcholinesterase inhibitors in patients with myasthenia gravis. Muscle Nerve 37: 300-307. [Crossref]

22. Seldin M, Alkhairy O, Willcox N (2015) Genome-wide association study of late-onset myasthenia gravis: Confirmation of TNFRSF11A and identification of ZBTB10 and three distinct HLA associations. Mol Med [serial online]. 21: 769-781. Available from: ScopusÂ ${ }^{\circledR}$, Ipswich, MA. Accessed September 3, 2016.

23. Barbaud A, Carlander B, Pages M (2016) Late onset forms of myasthenia gravis Comparison with early-onset myasthenia gravis. Revue Neurologique [serial online] 162: 990-996. Available from: Science Citation Index, Ipswich, MA. Accessed September 15, 2016

24. Guptill JG, Sanders DB, Eboli A (2011) Anti MuSK antibody myasthenia gravis, clinical findings and response to treatment in two large cohorts. Muscle Nerve 44: 467484. [Crossref]

25. ZivkoviÄ SA, Clemens PR, Lacomis D (2012) Characteristics of late-onset myasthenia gravis. J Neurol 259: 2167-2171. [Crossref]

26. Hellmann MA, Mosberg-Galili R, Steiner I (2013) Myasthenia gravis in the elderly. $J$ Neurol Sci 325: 1-5. [Crossref]

27. Suzuki S, Utsugisawa Y, Nagane T, Satoh T, Kuwana M (2011) Clinical and immunological difference between early and late onset myasthenia gravis in Japan. $J$ Neurol Neuroimunol 230: 148-152. [Crossref]

28. Mainaol AH, Elsois A, Lorecent AR (2012) Late onset myasthenia gravis is associated with HLA DRB1*15:01, in the Norwegian population. Plus One 8: 1932-2053. [Crossref]

29. Hellmann M, Mosberg-Galili R, Steiner I (2013) Myasthenia gravis in the elderly. $J$ Neurol Sci [serial online]. 325: 1-5. Available from: ScopusÂ ${ }^{\circledR}$, Ipswich, MA. Accessed September 15, 2016

30. Wolfe G, Kaminski H, Cutter G, et al. Randomized Trial of Thymectomy in Myasthenia Gravis. NEJM serial online]. 375: 511-522. Available from: MEDLINE, Ipswich, MA. Accessed September 3, 2016.

31. Nishikawa N, Nagai M, Nomoto M, et al. (2015) Treatment of Myasthenia Gravis in Patients with Elderly Onset at Advanced Age. Japanese Clin Med [serial online]. 6 :913. Available from: MEDLINE, Ipswich, MA. Accessed September 3, 2016.

32. Kapinas K, Kimiskidis V, Kazis A, Kokkas B, Tsolaki M, et al. Myasthenia gravis correlation of age with clinical course and anti-AChR antibody levels. Int $J$ Immunopathol Pharmacol [serial online]. 12: 127-131. Available from: Science Citation Index, Ipswich, MA. Accessed September 15, 2016

Copyright: (C2018 Porras-Yaurivilca AL. This is an open-access article distributed under the terms of the Creative Commons Attribution License, which permits unrestricted use, distribution, and reproduction in any medium, provided the original author and source are credited. 